\title{
A Systematic Review and Data Analysis on the Epidemiology of Tuberculosis and Diabetes Mellitus
}

\author{
Garima Singh ${ }^{1}$, Akhil Janardhanan ${ }^{2}$, Shweta Sharma ${ }^{3}$, Bharti Vyas ${ }^{1}$, Shakir Ali $^{3}$, Mymoona Akhter ${ }^{4}$ \\ ${ }^{1}$ Department of Bioinformatics, School of Interdisciplinary Sciences, Jamia Hamdard, \\ New Delhi, India \\ ${ }^{2}$ Department of Computational Biology and Bioinformatics, University of Kerala, Trivandrum, Kerela, India \\ ${ }^{3}$ Department of Biochemistry, School of Chemical and Biological Sciences, Jamia Hamdard, \\ New Delhi, India \\ ${ }^{4}$ Department of Pharmaceutical Chemistry, School of Pharmaceutical Education and Research, Jamia Hamdard, New \\ Delhi, India
}

\begin{abstract}
Objectives: This study aims to analyze epidemiological data that diabetes leads to increased susceptibility to initial tuberculosis infection or if diabetes leads to increased progression from latent tuberculosis to active tuberculosis.

Methods: A simplified MEDLINE search method has been used in this study. The PubMed's Clinical Queries, which have "research methodology filters" to study the patterns, causes, and clinical effects of health and the disease conditions in defined populations, were explored and extracted.

Results: Using the keyword-based queries in PubMed/ Medline and Embase, 38 relevant published studies were collected from 1970 to 2020. Published studies from the literature search were combined with the observational studies of summary statistics which can further be used for clinical trials.

Conclusion: The high prevalence of diabetes is a factor that increases the incidence of tuberculosis and is considered in the fight against tuberculosis worldwide. There have been many significant recent advances in knowledge regarding diabetes-associated tuberculosis's epidemiology, management, and control. J Microbiol Infect Dis 2021; 11(4):191-200.
\end{abstract}

Keywords: Mycobacterium tuberculosis, Diabetes, Epidemiology, Review analysis

\section{INTRODUCTION}

The relation between tuberculosis and diabetes has been known for centuries. Presently, tuberculosis prevalence remarkably decreased in high-income countries. However, occurrence remains high in countries with high rates of diabetes, the high commonness of malnutrition, and mass living conditions or insufficient tuberculosis control infrastructure. In addition, many studies showed that diabetes is an important risk factor for tuberculosis and might affect the disease presentation and treatment response.

Moreover, tuberculosis might induce glucose intolerance and inflaming glycaemic control in people with diabetes. Also, the drugs used in treating tuberculosis disease interact with oral anti-diabetic drugs [1]. In recent years, many important advances have been made in elucidating the epidemiology of diabetesrelated tuberculosis and in methods to diagnose, treat, and prevent tuberculosis and reduce mortality in tuberculosis/diabetes coinfected patients.

In sub-Saharan Africa, tuberculosis is a serious problem and is the leading cause of death in diabetic people. Diabetes is one of the paramount factors in the development of active and latent tuberculosis. Drug-resistant tuberculosis is a severe yet unsolved public 
health problem, especially in Southeast Asia, Africa. This study attempted to evaluate this primary source of information from where we collected already available data of the patients. This paper highlights the related findings in tuberculosis and diabetes co-infection epidemic, particularly globally.

Past studies are important in order to understand the present study. An early study reported in Germany in the year 1950s, where diabetic patients with tuberculosis treatment have been evaluated, after the completion of post-treatment of prophylaxis with isoniazid for 6-24 months, reported the lower rates of repetition in the intervention group [2]. Early studies showed that glucose intolerance occurs in the background of tuberculosis without diabetes when changes in carbohydrate metabolism during the primary attack of tuberculosis with enhanced insulin secretion and signs of respective insulin deficiency with persistent hyperglycemia as a forerunner to severe diabetes. Some specific tuberculosis studies showed the production of anti-insulin antibodies in mice, and mycobacterial proteins directly cause insulitis, hyperglycemia, and diabetes, which predict mainly autoimmune destruction of pancreatic islets in Type-1 diabetes.

In the previous, the studies conducted were expensive as well as time-consuming. These studies were problematic because of the absence of randomized details regarding interventions. Some studies showed several developments of severe diabetes in patients with pulmonary tuberculosis, relatively increased in the disease duration with deficiency associated with the higher secretary function of the insular pancreatic apparatus, which decreased its functional reserves [3]. In 1995, a study reported that pulmonary tuberculosis might predispose to glucose intolerance or endocrine abnormality [4]. It was found that $49 \%$ of the cases with pulmonary tuberculosis had bad glucose intolerance, which indicated that the patients with pulmonary tuberculosis should be screened positive with glucose intolerance.

Countries have different control strategies for tuberculosis. For example, a survey of State Tuberculosis Control Laws and Regulations showed that the US states differ in their approach to controlling tuberculosis [5]. The major goal of tuberculosis programs is to eliminate tuberculosis by appropriately treating persons infected with tuberculosis, safeguard the confidentiality and civil liberties of the persons who have tuberculosis, and protect them from unlawful discrimination because of their disease. Keeping in mind the epidemiology co-existence of tuberculosis/diabetes, we have carried out the data analysis of the two diseases.

\section{METHODS}

\section{Search strategy}

The information was retrieved from the literature sources using specific PubMed/MEDLINE and Embase queries. The search results were not limited to abstracts published in English. The abstracts were downloaded for further information extraction, and the full articles were studied that met the inclusion criteria. The articles retrieved using different queries are tabulated in tables (Table 1).

After searching the published relevant articles, population-based studies reporting the incidence and prevalence of tuberculosis and diabetes were evaluated. Studies were considered population-based if they employed a sampling method meant to represent the entire population and were completed in a defined geographical area of known population size. The studies were excluded if they did not provide an estimation of incidence or prevalence and the collected data before 1997 and whether the study reported non-original data (i.e., reviews, letters, or editorials).

\section{Data collection}

For this epidemiological study with respect to data analysis, PubMed/Medline and Embase (Excerpta Medica Database) were searched to identify all relevant published data from 1970 to 2020 on the prevalence of "Mycobacterium tuberculosis" pathogen for causing tuberculosis in patients with diabetes and vice versa worldwide. The search strategy included "tuberculosis", "diabetes", "anti-tuberculosis", "hyperglycemia", "epidemiology" and "data analysis".

A total of 2,304 studies from MEDLINE and EMBASE were collected, which includes all the search strategies to fulfill our research criteria. Duplicated articles, studies without population-based or incidence data were excluded, studies with abstracts only were 
excluded, only full-text articles were scanned. Thus, we finally included thirty-eight studies related to our study.

\section{Data Extraction}

Data extraction was done with each adult population-based cohort study published from 1997 till 2020, where prevalence could be calculated from available data and then taken for consideration. We have excluded the studies that presented estimation based on small subsets of a population, e.g., hospital outpatient, clinical patients. We did not exclude the selected populations that were considered to be illustrated in the general population of a particular geographical area. A complete list of inclusion and exclusion criteria is given in (Figure 1). After reading the articles, the information was extracted from the publications based on the year, country, author, time frame, age range, diagnostic criteria, data source, prevalence date, number of cases, gender, population size, and the potential replicating of data.

\section{Data Analysis}

It was used a graphical plot to show results from individual studies based on year-wise analysis and provide graphical information about estimates of the statistical significance of the cited publications (Figure 2).

Table 1. Number of citations retrieved using the various search terms

\begin{tabular}{lc}
\hline $\begin{array}{l}\text { PubMed/MEDLINE and Embase } \\
\text { Search Query }\end{array}$ & $\begin{array}{c}\text { Number of } \\
\text { abstracts }\end{array}$ \\
\hline ((Tuberculosis) AND (Diabetes)) & 1941 \\
$\begin{array}{l}\text { ((Pharmacokinetics) AND (anti- } \\
\text { tuberculosis) AND (enzymes)) }\end{array}$ & 69 \\
$\begin{array}{l}\text { ((Tuberculosis therapy) AND } \\
\text { (Diabetes) AND (hyperglycemia)) }\end{array}$ & 168 \\
$\begin{array}{l}\text { (((Tuberculosis) AND (Diabetes) } \\
\text { AND (hyperglycemia) AND } \\
\text { (epidemiology))) }\end{array}$ & 126 \\
\hline
\end{tabular}

\section{RESULTS}

The data of tuberculosis/diabetes was combined yearly in a list (Table 2).
Table 2. Tuberculosis and Diabetes stratified yearly since the year 1997 to 2020 .

\begin{tabular}{|c|c|c|}
\hline $\begin{array}{l}\text { Year of } \\
\text { Publication }\end{array}$ & $\begin{array}{l}\text { Sum of citation } \\
\text { per year }\end{array}$ & $\begin{array}{c}\text { Mean citation for } \\
\text { each year }\end{array}$ \\
\hline 1997 & 1263 & 1263 \\
\hline 2005 & 69 & 69 \\
\hline 2006 & 258 & 258 \\
\hline 2007 & 489 & 244.5 \\
\hline 2008 & 1519 & 1519 \\
\hline 2009 & 189 & 189 \\
\hline 2010 & 622 & 311 \\
\hline 2011 & 108 & 108 \\
\hline 2012 & 220 & 73.3 \\
\hline 2013 & 245 & 61.25 \\
\hline 2014 & 120 & 30 \\
\hline 2015 & 40 & 13.3 \\
\hline 2016 & 95 & 31.6 \\
\hline 2017 & 328 & 54.6 \\
\hline 2018 & 9 & 9 \\
\hline 2019 & 40 & 13.3 \\
\hline 2020 & 6 & 6 \\
\hline
\end{tabular}

Calculations:

Score $=($ Sum of citations of publications/Number of publications)/number of years passed.

Incidence studies of tuberculosis with known blood sugar status:

The studies used incidence based on the hospital or clinical records, administrative databases, and/or surveys of physicians to estimate incidence within the geographical boundaries of their service area from the literature search. The major parts of these studies were prevalence estimates based on systematic reviews.

\section{Prevalence studies}


In total, 38 studies were examined for the prevalence of tuberculosis with diabetes. Eight studies were from the USA, eight studies China, four studies Korea, thirteen studies different Asian countries, two studies African countries, and three from global population studies included in this study (Table 3).

\section{Risk factors for tuberculosis/diabetes comorbidity}

The risk factors associated with tuberculosis/diabetes comorbidity were heterogeneous. The studies used different measures to analyze the factors coalition with the disease. Some studies reported tuberculosis/diabetes risk consortium factors using odds ratio, relative risk ratio, or hazard ratio. Conversely, other studies did not report either interrelation or risk factors for tuberculosis/diabetes co-existence. This might be due to that the studies did not have enough sample size, and almost all studies mentioned neither in their objective nor in the controlled part about the risk factors of tuberculosis/diabetes comorbidity.

Numerous studies concluded that both males and females [6-10] of all ages were at high risk for tuberculosis/diabetes comorbidity regarding socio-demographic and socioeconomic factors. At the same time, men were more likely to develop tuberculosis/diabetes comorbidity than women [11]. Furthermore, 22 studies reported that older aged people are mostly the ones who are at increased risk of tuberculosis/diabetes comorbidity [8-28].

When we look at the behavioral risk factors, illegal drug consumption and inactive lifestyle were among the most reported behavioral factors related to tuberculosis/diabetes comorbidity [11,29]. In addition, smoking [7] and being an alcoholic [15] are reported as increased risk factors for tuberculosis/diabetes co-existence. On the other hand, practicing a good active lifestyle and various frequent outdoor activities were less behavioral risk factors for tuberculosis/diabetes comorbidity [19].

Body mass index (BMI), whether higher or lower, human immune-deficiency virus (HIV)

\section{DISCUSSION}

Diabetes is the most potent risk factor for tuberculosis. Diabetes co-infection with tuberculosis collate with diabetes hyperglycemia, anatomy of which recorded in diabetes prevalence countries. March 2019, WHO declared a new intent to end tuberculosis in the world by 2030. In many developing countries where healthcare resources are limited, diabetes and tuberculosis comorbidity is a public health concern. co-infection, bodyweight loss or gain in body weight, hypertension, and hyperglycemia were reported as associated factors for tuberculosis/diabetes comorbidity $[7,8,11,17,18]$. Pre-existence as well as long duration of diabetes [8], poor glycemic control reported during tuberculosis diagnosis, patients with liver cirrhosis [7], and high blood pressure [17] were identified as some of the main reasons for increased risk factors for the development of tuberculosis/diabetes comorbidity. Diabetes with positive [11] and negative HIV conditions was linked and increased risk factors for tuberculosis/diabetes comorbid condition, whereas HIV co-infection and malnutrition were also suggested as lowrisk factors for tuberculosis/diabetes comorbidity [10]. On the other hand, being extra-pulmonary tuberculosis (EPTB) case was reported as a low-risk factor for tuberculosis/diabetes comorbidity [28].

In some studies showed that direct contact with the tuberculosis patient within the family was reported as an increased risk factor for tuberculosis/diabetes comorbidity. Being a prisoner was associated with tuberculosis/diabetes comorbidity [11]. TBDM patients were more expected to die from tuberculosis/diabetes comorbidity [28]. On the other hand, retained in some institutions (prisons, shelters, orphanages, and psychiatric hospitals) were reported as low-risk factors for tuberculosis/diabetes comorbidity [28].

According to World Health Organisation (WHO) treatment guidelines, tuberculosis suspected patients should be treated with a four-drug rule regimen, which is a breakup in a starting two months intensive phase, i.e., isoniazid, rifampicin, ethambutol, pyrazinamide, and a four months two drugs continuing phase (isoniazid and rifampicin). In comparison, the patients with poor response and evidence of drug resistance are advised quickly to initiate treatment with second-line drugs. In this study, potential interactions with second-line drugs are not reported. 


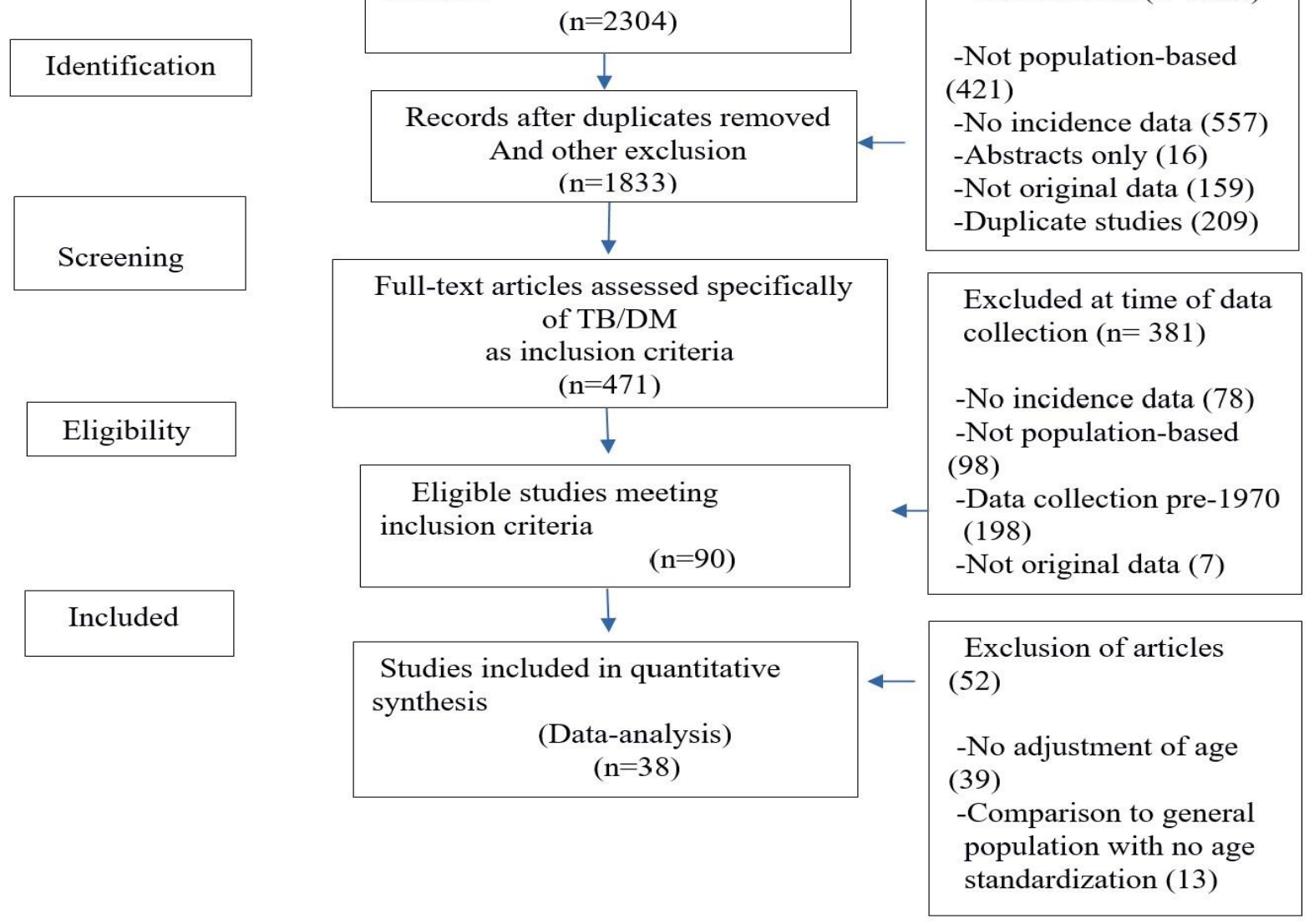

Figure 1. Study selection criteria via flow chart.

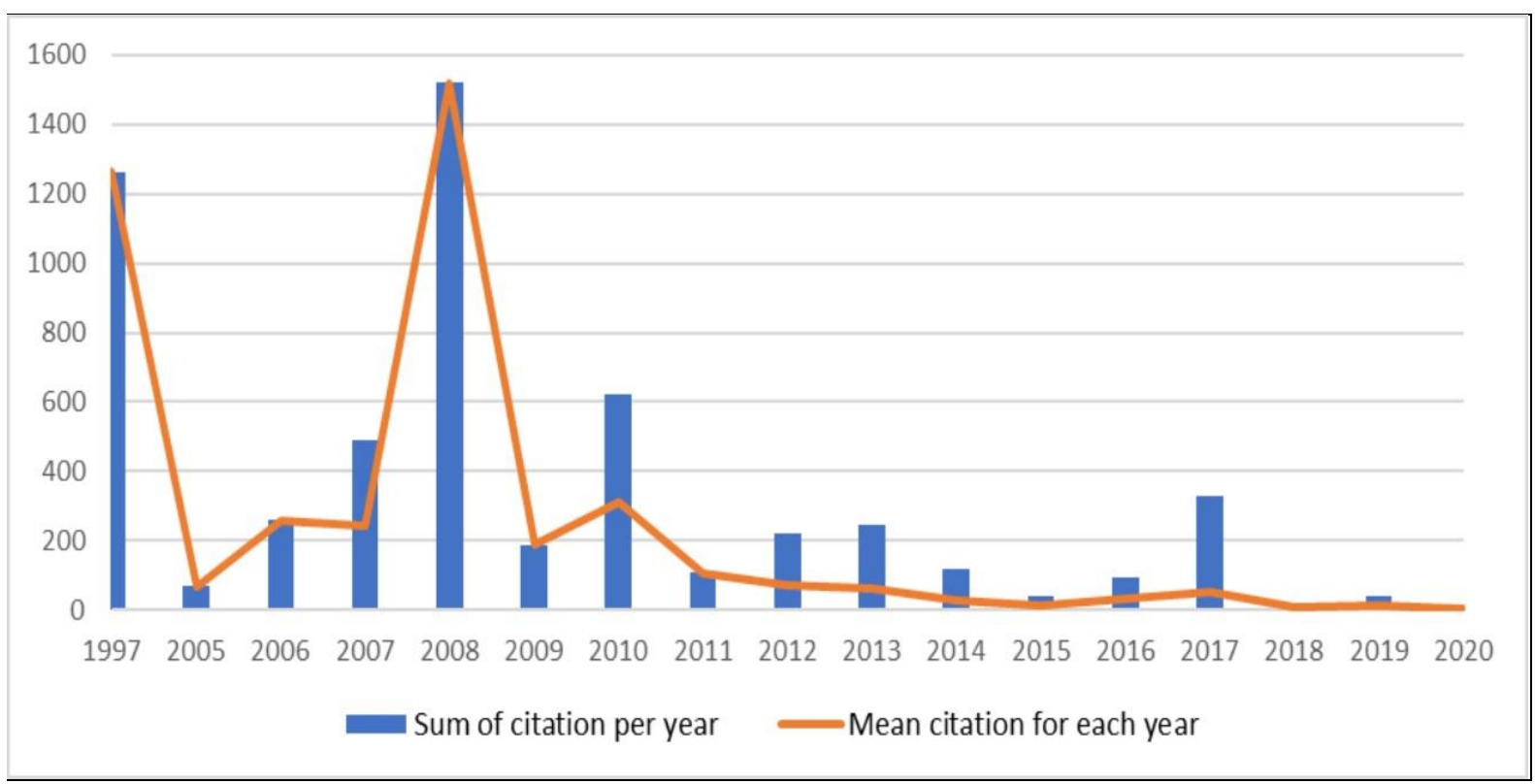

Figure 2. Year-wise citations of Tuberculosis and Diabetes cases (1997-2020) 
Table 3. Studies were examined for the prevalence of TB with DM.

\begin{tabular}{|c|c|c|c|c|c|c|c|c|}
\hline S.No & Author & $\begin{array}{l}\text { Publication } \\
\text { year, country }\end{array}$ & $\begin{array}{l}\text { Time } \\
\text { frame } \\
\text { and } \\
\text { age } \\
\text { range }\end{array}$ & $\begin{array}{l}\text { Inclusion } \\
\text { Criteria }\end{array}$ & $\begin{array}{c}\text { Prevalence } \\
\text { date }\end{array}$ & $\begin{array}{l}\text { Number of } \\
\text { cases }\end{array}$ & Gender & $\begin{array}{l}\text { Population } \\
\text { size } \\
\text { (Millions) }\end{array}$ \\
\hline 1. & $\begin{array}{l}\text { Ariel Pablos- } \\
\text { Mendez }\end{array}$ & $\begin{array}{c}1997, \\
\text { California, USA }\end{array}$ & $\begin{array}{l}1997, \\
\text { all ages }\end{array}$ & $\begin{array}{l}\text { Hospital, clinic } \\
\text { chart review }\end{array}$ & 1991 & 5290 & Both & 32.2 \\
\hline 2. & $\begin{array}{l}\text { Lawrence } \\
\text { Broxmeyer }\end{array}$ & 2005, US & \begin{tabular}{|}
$1934-$ \\
2004, \\
all ages
\end{tabular} & Review & 1934-2004 & $\begin{array}{c}\text { not } \\
\text { specified }\end{array}$ & Both & 295.5 \\
\hline 3. & Nijland et al & $\begin{array}{l}\text { 2006, Jakarta, } \\
\text { Indonesia }\end{array}$ & $\begin{array}{l}2006, \\
\text { all ages }\end{array}$ & $\begin{array}{l}\text { clinic chart } \\
\text { review }\end{array}$ & 2006 & 36 & Both & 229.3 \\
\hline 4. & $\begin{array}{c}\text { Alisjahbana } \\
\text { et al }\end{array}$ & $\begin{array}{l}\text { 2007, } \\
\text { Indonesia }\end{array}$ & $\begin{array}{l}2000- \\
2005, \\
\text { all ages }\end{array}$ & $\begin{array}{l}\text { Hospital, clinic } \\
\text { chart review }\end{array}$ & $2000-2005$ & 737 & Both & 232.4 \\
\hline 5. & Nigel Unwin & 2007, India & $\begin{array}{l}2000, \\
\text { all ages }\end{array}$ & $\begin{array}{c}\text { "administrative } \\
\text { data }\end{array}$ & 2000 & 116000 & Both & 1183.2 \\
\hline 6. & $\begin{array}{l}\text { Christie Y. } \\
\text { Jeon }\end{array}$ & 2008, (USA) & $\begin{array}{l}1965- \\
2007\end{array}$ & $\begin{array}{l}\text { clinic chart } \\
\text { review }\end{array}$ & $1965-2007$ & 17698 & Both & 304.1 \\
\hline 7. & NA & 2009 , global & $\begin{array}{l}2009, \\
\text { all ages }\end{array}$ & $\begin{array}{l}\text { clinic chart } \\
\text { review }\end{array}$ & 2009 & 36 & Both & $\begin{array}{c}\text { Not } \\
\text { specified }\end{array}$ \\
\hline 8. & $\begin{array}{l}\text { Rovina } \\
\text { Ruslami }\end{array}$ & $\begin{array}{l}\text { 2010, } \\
\text { Indonesia }\end{array}$ & $\begin{array}{l}2009, \\
\text { all ages }\end{array}$ & $\begin{array}{c}\text { administrative } \\
\text { database, chart } \\
\text { review }\end{array}$ & 2009 & 36 & Both & 241.8 \\
\hline 9. & $\begin{array}{c}\text { A. D. Harries } \\
\text { et al }\end{array}$ & $\begin{array}{l}\text { 2010, Paris, } \\
\text { France }\end{array}$ & $\begin{array}{l}\text { May } \\
2009- \\
\text { August } \\
2009\end{array}$ & $\begin{array}{l}\text { age-adjusted, } \\
\text { quantitative, } \\
\text { observational } \\
\text { studies }\end{array}$ & $\begin{array}{l}\text { May 2009- } \\
\text { August } \\
2009\end{array}$ & $\begin{array}{c}\text { not } \\
\text { specified }\end{array}$ & Both & 104.6 \\
\hline 10. & $\begin{array}{c}\text { Christopher } \\
\text { Dye }\end{array}$ & $\begin{array}{c}\text { 2011, India and } \\
\text { Korea }\end{array}$ & $\begin{array}{l}1998- \\
2008, \\
\text { all ages }\end{array}$ & $\begin{array}{l}\text { administrative } \\
\text { data }\end{array}$ & 1998-2008 & $\begin{array}{c}\text { not } \\
\text { specified }\end{array}$ & Both & 1250.3 \\
\hline 11. & $\begin{array}{l}\text { Claudia } \\
\text { Caroline } \\
\text { Dobler }\end{array}$ & 2012, Australia & $\begin{array}{l}2001- \\
2006, \\
\text { all ages }\end{array}$ & $\begin{array}{l}\text { administrative } \\
\text { database, } \\
\text { clinical chart }\end{array}$ & 2001-2006 & 6276 & Both & 19. 855283 \\
\hline 12. & $\begin{array}{l}\text { Diana I. } \\
\text { Gomez }\end{array}$ & $\begin{array}{l}\text { 2012, South } \\
\text { Texas }\end{array}$ & $\begin{array}{l}2012, \\
\text { all ages }\end{array}$ & $\begin{array}{l}\text { clinical chart, } \\
\text { review }\end{array}$ & 2012 & $\begin{array}{c}\text { not } \\
\text { specified }\end{array}$ & Both & 313.9 \\
\hline 13. & $\begin{array}{l}\text { Liaqat Ali } \\
\text { Chaudhry }\end{array}$ & $\begin{array}{l}\text { 2012, Saudi } \\
\text { Arabia }\end{array}$ & $\begin{array}{l}2003- \\
2010, \\
\text { all ages }\end{array}$ & $\begin{array}{l}\text { clinical chart, } \\
\text { Review }\end{array}$ & $\begin{array}{l}2003 \text { and } \\
2010 .\end{array}$ & 1388 & Both & 29.2 \\
\hline 14. & $\begin{array}{l}\text { Lyudmila } \\
\text { Boyanova }\end{array}$ & $\begin{array}{c}\text { 2013, } \\
\text { Bulgaria(USA) }\end{array}$ & $\begin{array}{l}2009- \\
2013, \\
\text { all ages }\end{array}$ & Review & $2009-2013$ & $\begin{array}{c}\text { not } \\
\text { specified }\end{array}$ & Both & 7.28 \\
\hline 15. & Aylin Babalik & $\begin{array}{c}\text { 2013, Istanbul, } \\
\text { Turkey }\end{array}$ & $\begin{array}{l}2013, \\
\text { all ages }\end{array}$ & $\begin{array}{l}\text { clinic chart } \\
\text { review }\end{array}$ & 2013 & 70 & Both & 13.658 \\
\hline 16. & V. & 2013, South & $\begin{array}{l}2007- \\
2013\end{array}$ & Hospital, clinic & $2007-2013$ & 245 & Both & 210 \\
\hline
\end{tabular}




\begin{tabular}{|c|c|c|c|c|c|c|c|c|}
\hline & Viswanathan & India & all ages & chart review & & & & \\
\hline 17. & $\begin{array}{c}\text { Boillat- } \\
\text { Blanco et al. }\end{array}$ & $\begin{array}{l}\text { 2013,Kinondoni } \\
\text { District, Taiwan }\end{array}$ & $\begin{array}{l}2013, \\
\text { all ages }\end{array}$ & $\begin{array}{l}\text { Hospital, clinic } \\
\text { chart review }\end{array}$ & 2013 & 250 & Both & 1.3574 \\
\hline 18. & Fengling Mi & $\begin{array}{l}\text { 2014, Beijing, } \\
\text { China }\end{array}$ & $\begin{array}{l}2011- \\
2012, \\
\text { all ages }\end{array}$ & $\begin{array}{l}\text { clinic chart } \\
\text { review }\end{array}$ & $\begin{array}{l}2011-2012, \\
\text { all ages }\end{array}$ & 621 & Both & 13 \\
\hline 19. & $\begin{array}{c}\text { M. J. Magee } \\
\text { et al. }\end{array}$ & 2014, Georgia & $\begin{array}{l}\text { 2009- } \\
2012, \\
\text { all ages }\end{array}$ & $\begin{array}{c}\text { administrative } \\
\text { database, clinic } \\
\text { chart review }\end{array}$ & 2009-2012 & 1325 & Both & 10.1 \\
\hline 20. & $\begin{array}{c}\text { M. J. Chang } \\
\text { et al }\end{array}$ & 2014, Korea & $\begin{array}{l}2014, \\
\text { all ages }\end{array}$ & $\begin{array}{c}\text { administrative } \\
\text { database, clinic } \\
\text { chart review }\end{array}$ & 2014 & 54 & Both & 50.7 \\
\hline 21. & $\begin{array}{l}\text { Marit Eika } \\
\text { Jørgensen }\end{array}$ & 2014, Denmark & $\begin{array}{l}\text { 1990- } \\
2013, \\
\text { all ages }\end{array}$ & $\begin{array}{l}\text { clinical trial } \\
\text { database }\end{array}$ & $1990-2013$ & 4959 & Both & 5.6 \\
\hline 22. & $\begin{array}{l}\text { Yu-Cheng } \\
\text { Chen }\end{array}$ & 2015, Taiwan & $\begin{array}{l}2000- \\
2010, \\
\text { all ages }\end{array}$ & $\begin{array}{c}\text { hospital/clinic } \\
\text { chart review, } \\
\text { administrative } \\
\text { data }\end{array}$ & 2000-2010 & 2492 & Both & 1 \\
\hline 23. & $\begin{array}{l}\text { Saurabh } \\
\text { Mehta }\end{array}$ & $\begin{array}{l}\text { 2015, Andhra } \\
\text { Pradesh, India }\end{array}$ & $\begin{array}{l}2012- \\
2013, \\
\text { all ages }\end{array}$ & $\begin{array}{l}\text { clinic chart } \\
\text { review, } \\
\text { administrative } \\
\text { data }\end{array}$ & 2013 & 304 & Both & 276 \\
\hline 24. & Wang & $\begin{array}{l}\text { 2015, Taiwan } \\
\text { (China) }\end{array}$ & $\begin{array}{l}\text { 1996- } \\
2010, \\
\text { all ages }\end{array}$ & $\begin{array}{c}\text { Mailed survey, } \\
\text { hospital/clinic } \\
\text { chart review, } \\
\text { administrative } \\
\text { data }\end{array}$ & 2010 & 151571 & Both & $234,920,000$ \\
\hline 25. & $\begin{array}{l}\text { Nathella } \\
\text { Pavan } \\
\text { Kumar }\end{array}$ & 2016, India & $\begin{array}{c}2016, \\
\text { all ages }\end{array}$ & $\begin{array}{l}\text { Clinical charts, } \\
\text { Graphs plot" }\end{array}$ & 2016 & 57 & Both & 1324.5 \\
\hline 26. & $\begin{array}{l}\text { Malabika } \\
\text { Sarker }\end{array}$ & $\begin{array}{c}2016, \\
\text { Bangladesh }\end{array}$ & $\begin{array}{l}2016, \\
\text { all ages }\end{array}$ & $\begin{array}{l}\text { Hospital, clinic } \\
\text { chart review }\end{array}$ & 2016 & 1910 & Both & 158 \\
\hline 27. & Hsien-Ho Lin & 2016, Taiwan & $\begin{array}{l}2016, \\
\text { all ages }\end{array}$ & $\begin{array}{l}\text { administrative } \\
\text { database }\end{array}$ & 2016 & 38263 & Both & 23.6182 \\
\hline 28. & $\begin{array}{l}\text { Yu-Cheng } \\
\text { Chen }\end{array}$ & 2017, Taiwan & $\begin{array}{l}2000- \\
2010, \\
\text { all ages }\end{array}$ & $\begin{array}{c}\text { Hospital, } \\
\text { administrative } \\
\text { database, clinic } \\
\text { chart review }\end{array}$ & $2000-2010$ & 9833 & Both & 23.6745460 \\
\hline 29. & Zheng et al & 2017, China & 2017 & $\begin{array}{l}\text { administrative } \\
\text { database, clinic } \\
\text { chart review }\end{array}$ & 2017 & & Both & 1386.4 \\
\hline 30. & $\begin{array}{l}\text { Rami H. Al- } \\
\text { Rifai }\end{array}$ & 2017, USA & $\begin{array}{l}1945- \\
2015, \\
\text { all ages }\end{array}$ & $\begin{array}{l}\text { Database } \\
\text { review }\end{array}$ & $1945-2015$ & $5,84,72,375$ & Both & 325.1 \\
\hline 31. & $\begin{array}{l}\text { Mahteme } \\
\text { Haile }\end{array}$ & $\begin{array}{c}\text { 2017, Asia, } \\
\text { North America, }\end{array}$ & $\begin{array}{l}1900- \\
2016\end{array}$ & $\begin{array}{l}\text { Database } \\
\text { review }\end{array}$ & $1900-2015$ & 1845 & Both & $\begin{array}{c}\text { Not } \\
\text { specified }\end{array}$ \\
\hline
\end{tabular}




\begin{tabular}{|c|c|c|c|c|c|c|c|c|}
\hline & Workneh & Oceania & all ages & & & & & \\
\hline 32. & $\begin{array}{l}\text { Hemant } \\
\text { Deepak } \\
\text { Shewade }\end{array}$ & 2017, USA & $\begin{array}{c}1996- \\
2017, \\
\text { all ages }\end{array}$ & $\begin{array}{l}\text { electronic } \\
\text { databases }\end{array}$ & $1996-2017$ & 2326 & Both & 325.1 \\
\hline 33. & $\begin{array}{l}\text { Song Yee } \\
\text { Kim }\end{array}$ & $\begin{array}{l}\text { 2017, Seoul, } \\
\text { South Korea }\end{array}$ & $\begin{array}{l}2010- \\
2012, \\
\text { all ages }\end{array}$ & $\begin{array}{l}\text { Patients } \\
\text { Registry }\end{array}$ & $2010-2012$ & 1044 & Both & 9.78 \\
\hline 34. & $\begin{array}{c}\text { Fasil } \\
\text { Wagnew }\end{array}$ & $\begin{array}{l}\text { 2018, African } \\
\text { and Asian } \\
\text { countries }\end{array}$ & $\begin{array}{c}1980- \\
2017, \\
\text { all ages }\end{array}$ & $\begin{array}{l}\text { clinic chart } \\
\text { review, } \\
\text { administrative } \\
\text { data }\end{array}$ & $1980-2017$ & 23068 & Both & $\begin{array}{c}\text { Not } \\
\text { specified }\end{array}$ \\
\hline 35. & $\begin{array}{l}\text { Jean Joel } \\
\text { Bigna }\end{array}$ & Woldwide & $\begin{array}{c}1986- \\
2017, \\
\text { all ages }\end{array}$ & $\begin{array}{c}\text { Administrative } \\
\text { database, Plots }\end{array}$ & $\begin{array}{c}\text { Jan 1, } \\
\text { 1986, and } \\
\text { June 15, } \\
2017\end{array}$ & $2 \cdot 3$ million & Both & 230 \\
\hline 36. & Mtabho et al & 2019, Tanzania & $\begin{array}{c}2019, \\
\text { all ages }\end{array}$ & $\begin{array}{c}\text { hospital/clinic } \\
\text { chart review, } \\
\text { administrative } \\
\text { data }\end{array}$ & 2019 & 40 & $\begin{array}{l}\text { age } 18 \\
\text { years, } \\
\text { Both }\end{array}$ & 58.005463 \\
\hline 37. & J. E. Golub & 2019, Korea & $\begin{array}{l}1997- \\
2000, \\
\text { all ages }\end{array}$ & $\begin{array}{c}\text { hospital/clinic } \\
\text { chart review, } \\
\text { administrative } \\
\text { data }\end{array}$ & $1997-2000$ & 1267564 & Both & 5.126436 \\
\hline 38. & $\begin{array}{l}\text { Jianming } \\
\text { Wang }\end{array}$ & $\begin{array}{l}\text { 2020, Jiangsu } \\
\text { Province, } \\
\text { China }\end{array}$ & $\begin{array}{c}2016- \\
2018, \text { all } \\
\text { ages }\end{array}$ & $\begin{array}{c}\text { hospital/clinic } \\
\text { chart review, } \\
\text { administrative } \\
\text { data }\end{array}$ & $2016-2018$ & 14869 & Both & 14,393 \\
\hline
\end{tabular}

The prevalence of diabetes is estimated to increase from about 382 million in 2013 to around 592 million in 2030. In 2013 about 80\% of diabetes patients lived in developing countries where healthcare resources were limited. The more significant concern is that about $48 \%$ of the patients with diabetes were undiagnosed. Diabetes patients amid in lowincome countries are increasing in number; thus, tuberculosis/diabetes comorbidity could inevitably increase. This might hamper the WHO target to decrease global tuberculosis incidence by $90 \%$ or less than 10 cases per 100,000 populations in 2035 . Furthermore, it is more difficult for the world's long-term vision to eliminate tuberculosis as a public health concern by reducing tuberculosis incidence to less than 1 case per million of the population by 2050 . The study collected here has its pros and cons. This study included many studies covering almost all worldwide data, which is the pros of this study. Cons of this study are the exclusion of studies written in language rather than English. Inclusion study criteria which were taken here accommodated in the studies associated/risk factors of tuberculosis/diabetes comorbidity; therefore, the effect of excluding languages other than English written articles as of which findings of an empirical investigation grab for a variety of populations would be minimal. Some reported studies showed the population and gender of patients associated with tuberculosis and diabetes. The studies were observational studies and descriptive studies. Thus, we have used prevalence and incidence rate as our total effort to relate it with what the reviewed articles reported worldwide. This study is combined with the data analysis of summary statistics which were frequently used in citations. Our main objective was to understand the global epidemiology of tuberculosis/diabetes comorbidity having a less moderate risk of bias in a more comprehensive manner.

Conclusion: However, although they have appeared in limited numbers and are supportive, they have come at what, for 
diabetics, has been a painfully slow rate. Latent tuberculosis infection occurs when tubercle bacilli are in the body; the immune system keeps them under control. Nevertheless, suppose the immune system weakens in people with high diabetes ubiquity. In that case, tuberculosis can become active, and during its active state, it can cause the death of tissue in the organs they infect. We collected the evidence from an increased risk of tuberculosis patients with diabetes despite the variations in study design, with joint founders to control severe disease and control it with the exposure of its outcome of the burden of tuberculosis geographical boundaries. Data analyses which showed the human studies of different age groups and other factors are consistent with rising information on the biological mechanisms which may affect the host immune response to tuberculosis, including hyperglycaemic conditions. Among various factors that contributed to internet information exploring the data are: using a web browser and databases connected to web browser front ends.

\section{ACKNOWLEDGMENTS}

The authors are thankful to the Department of Biotechnology, Ministry of Science \& Technology, Government of India for the Bioinformatics infrastructure facility at Jamia Hamdard under BTIS-Net program. One of the authors, Ms. Garima Singh, acknowledges the fellowship grant from the Ministry of Social Justice and Empowerment, New Delhi.

Declaration of conflicting interest: The author(s) declare no potential conflicts of interest concerning this article's research, authorship, and/or publication.

Financial disclosure: No financial support was received for this study

\section{REFERENCES}

1. Dooley KE, Chaisson RE. Tuberculosis and diabetes mellitus: convergence of two epidemics. Lancet Infect Dis 2009; 9(12):737746.

2. Baghaei $P$, Marjani M, Javanmard $P$, Tabarsi $P$, Masjedi MR. Diabetes mellitus and tuberculosis facts and controversies. J Diabetes Metab Disord 2013;12(1):58.
3. Karachunskiĭ MA, Balabolkin MI, Beglarian NR. [Changes in carbohydrate metabolism in patients with tuberculosis]. Vestn Ross Akad Med Nauk 1995;(7):18-21.

4. Jawad F, Shera AS, Memon R, Ansari G. Glucose intolerance in pulmonary tuberculosis. JPMA J Pak Med Assoc 1995;45(9):237-238.

5. ACET. Tuberculosis Control Laws - United States, 1993 Recommendations of the Advisory Council for the Elimination of Tuberculosis (ACET).

6. Lin $\mathrm{Y}-\mathrm{H}$, Chen $\mathrm{C}-\mathrm{P}$, Chen $\mathrm{P}-\mathrm{Y}$, et al. Screening for pulmonary tuberculosis in type 2 diabetes elderly: a cross-sectional study in a community hospital. BMC Public Health 2015;15(1):3.

7. Suwanpimolkul G, Grinsdale JA, Jarlsberg LG, et al. Association between Diabetes Mellitus and Tuberculosis in United States-Born and ForeignBorn Populations in San Francisco. Caylà JA, ed. PLoS One 2014;9(12):e114442.

8. Workneh MH, Bjune GA, Yimer SA. Prevalence and Associated Factors of Diabetes Mellitus among Tuberculosis Patients in South-Eastern Amhara Region, Ethiopia: A Cross Sectional Study. Pai $\mathrm{M}$, ed. PLOS One 2016;11(1):e0147621.

9. Restrepo BI, Fisher-Hoch SP, Crespo JG, et al. Type 2 diabetes and tuberculosis in a dynamic bi-national border population. Epidemiol Infect 2007;135(3):483-491.

10. Delgado-Sánchez G, García-García L, Castellanos-Joya $M$, et al. Association of Pulmonary Tuberculosis and Diabetes in Mexico: Analysis of the National Tuberculosis Registry 2000-2012. DeRiemer K, ed. PLOS One 2015;10(6):e0129312.

11. Alavi SM, Khoshkhoy MM. Pulmonary tuberculosis and diabetes mellitus: Co-existence of both diseases in patients admitted in a teaching hospital in the southwest of Iran. Casp $\mathrm{J}$ Intern Med 2012;3(2):421-424.

12. Achanta S, Tekumalla RR, Jaju J, et al. Screening tuberculosis patients for diabetes in a tribal area in South India. Public Health Action 2013; 3(Suppl 1):S43-47.

13. Baghaei $P$, Tabarsi $P$, Marjani M, Moniri A, Masjedi MR. Screening for diabetes mellitus in tuberculosis patients in a referral center in Iran. Infect Dis 2015;47(7):472-476.

14. Dave $P$, Shah $A$, Chauhan $M$, et al. Screening patients with tuberculosis for diabetes mellitus in Gujarat, India. Public Health Action 2013;3(Suppl 1):S29-33.

15. Raghuraman $\mathrm{S}$, Vasudevan KP, Govindarajan S, Chinnakali P, Panigrahi KC. Prevalence of Diabetes Mellitus among 
Tuberculosis Patients in Urban Puducherry. North Am J Med Sci 2014;6(1):30-34.

16. Shidam UG, Roy G, Sahu SK, Kumar SV, Ananthanarayanan $\mathrm{PH}$. Screening for diabetes among presumptive tuberculosis patients at a tertiary care centre in Pondicherry, India. Int $\mathrm{J}$ Tuberc Lung Dis Off $\mathrm{J}$ Int Union Tuberc Lung Dis 2015;19(10):1163-1168.

17. Thapa, B., Paudel, R., Thapa, P., Shrestha, A., \& Poudyal, A. (2016). Prevalence of Diabetes among Tuberculosis Patients and Associated Risk Factors in Kathmandu Valley. SAARC J Tuberc Lung Dis HIVAIDS 122 20-27.

18. Viswanathan $\mathrm{V}$, Kumpatla $\mathrm{S}$, Aravindalochanan $\mathrm{V}$, et al. Prevalence of Diabetes and Pre-Diabetes and Associated Risk Factors among Tuberculosis Patients in India. Pai M, ed. PLoS One 2012;7(7):e41367.

19. Wang $Q$, Ma A, Han X, et al. Prevalence of Type 2 Diabetes among Newly Detected Pulmonary Tuberculosis Patients in China: A Community Based Cohort Study. Caylà JA, ed. PLoS One 2013;8(12):e82660.

20. Sulaiman SAS, Khan $\mathrm{AH}, \mathrm{Ahmad} \mathrm{N}$, lqubal MS, Muttalif AR, Hassali MA. Impact of Diabetes Mellitus on Treatment Outcomes of Tuberculosis Patients in Tertiary Care Setup. Am J Med Sci 2013;345(4):321-325.

21. Wu Z, Guo J, Huang Y, et al. Diabetes mellitus in patients with pulmonary tuberculosis in an aging population in Shanghai, China: Prevalence, clinical characteristics and outcomes. J Diabetes Complications 2016; 30(2):237-241.

22. Nair S, Kumari AK, Subramonianpillai J, et al. High prevalence of undiagnosed diabetes among tuberculosis patients in peripheral health facilities in Kerala. Public Health Action 2013;3(Suppl 1):S38-42.

23. MD Kottarath, $R$ Mavila, $V$ Achuthan, $S$ Nair - 2015. Prevalence of diabetes mellitus in tuberculosis patients: a hospital based study. Int J Res Med Sci 2015; 3(10):2810-2814.

24. Rao MSS, Shridhar M, Pavani K, V VE, Dass SM. Screening of tuberculosis in diabetic patients at a tertiary care hospital in Hyderabad. Indian J Microbiol Res 2015; 2(4):220-226.

25. Tiroro S. The Magnitude and Associated Factors of Tuberculosis among Diabetic Patients at Tikur Anbessa Specialized Teaching Hospital in Addis Ababa,Ethiopia. 2015. MPH Thesis. Addis Ababa University.

26. Rakotonirina J, Razanakoto $\mathrm{H}$, Rasolofomanana L, Razanakolona L. HIV prevalence and diabetes prevalence among tuberculosis patients in Antananarivo city: a descriptive study. Int $\mathrm{J}$ Res Med Sci 2014;2(3):834.

27. Castellanos-Joya M, Delgado-Sánchez G, Ferreyra-Reyes L, et al. Results of the implementation of a pilot model for the bidirectional screening and joint management of patients with pulmonary tuberculosis and diabetes mellitus in Mexico. Plos One 2014;9(9):e106961.

28. Reis-Santos B, Locatelli R, Horta BL, et al. Socio-demographic and clinical differences in subjects with tuberculosis with and without diabetes mellitus in Brazil--a multivariate analysis. PloS One 2013;8(4):e62604.

29. Baldé NM, Camara A, Camara LM, Diallo MM, Kaké A, Bah-Sow OY. Associated tuberculosis and diabetes in Conakry, Guinea: prevalence and clinical characteristics. Int $J$ Tuberc Lung Dis Off $\mathrm{J}$ Int Union Tuberc Lung Dis 2006;10(9):1036-1040. 